\title{
Models of the organisation of comprehensive care for children with ASD in the west
}

\begin{abstract}
The first Autistic societies were established in England and Denmark in 1962, followed by many other countries. Since that time there have been enormous strides in autism awareness and the development of medical, educational and social services for autistic children and their families. Current research has aimed to enhance our understanding of autism spectrum disorder, to inform both the practitioners and relevant governmental and non-governmental organisations about the necessity of creating comprehensive structures to support autistic individuals and their families and to contribute the ideas for the development appropriate services. As a review of all the available literature is beyond the scope of this paper, only a selection of the latest research studies relevant to the main trends in the development of the system of support is included. The article provides a brief review of the latest research in several key aspects of autism (such as: costs, prevalence, diagnosis, comorbids, etc.) that can inform the policy makers, specialists and parents about what can be done to improve the lives of autistic individuals
\end{abstract}

Keywords: autism, prevalence, biomarkers, diagnosis, comorbid conditions, educational provisions, heterogeneity, interventions
Volume 5 Issue I - 2018

\section{Olga Bogdashina}

University of Birmingham, European Institute of Child Education \& Psychology, UK

\section{Correspondence: Olga Bogdashina,Visiting Professor in Autism Studies (University of Birmingham), The European Institute of Child Education \& Psychology, Great Britain, UK, Email 07.olga@gmail.com}

Received: November 03, 2017 | Published: February 16, 2018
Abbreviations: $\mathrm{CPC}$, centers for desease control and prevention; ADDM, autism and developmental disabilities monitoring; PET, positron-emission tomography; MRI, magnetic resonance imaging; ASD, autism spectrum disorder

\section{Introduction}

As Roy Richard Grinker, ${ }^{1}$ an anthropologist and a father of an autistic girl, points out, despite autism being "a terrible, lifelong disorder", it is "a better time than ever to be autistic" because a person with this diagnosis has access to more services and opportunities than ever before. Nevertheless, there are still a lot of problems to be solved and questions to be answered. One of them (most important for the governmental organisations) is the economic effect of ASD.

\section{Costs}

Buescher $\mathrm{AV}$, et al. $^{2}$ estimate age-specific, direct, and lifetime societal economic costs, including indirect costs, associated with ASDs in the United Kingdom and the USA:

a. The cost of supporting an individual with an ASD and intellectual disability during his or her lifespan was $\$ 2.4$ million in the United States and $£ 1.5$ million (US \$2.2 million) in the United Kingdom. The cost of supporting an individual with an ASD without intellectual disability was $\$ 1.4$ million in the United States and $£ 0.92$ million (US \$1.4 million) in the United Kingdom.

b. The largest cost components for children were special education services and parental productivity loss. During adulthood, residential care of supportive living accommodation and individual productivity loss contributed the highest costs. Medical costs were much higher for adults than for children.

c. The authors, ${ }^{2}$ conclude that the substantial direct and indirect economic effect of ASDs emphasizes the need to continue to search for effective interventions that make best use of scarce societal resources. The distribution of economic effect across many different service systems raises questions about coordination of services and sectors. The enormous effects on families also warrant policy attention. With the early diagnosis and provision of the appropriate medical and educational services, the costs are likely to drop.

\section{Prevalence}

In order to calculate the necessary investments into medical, educational and social services, governmental medical organisations commission regular reports of the prevalence of autism in the country.

USA: In 2016, a report of prevalence of ASD, commissioned by the Centers for Desease Control and Prevention (CPC) was published in which the Autism and Developmental Disabilities Monitoring (ADDM) Network provides estimates of the prevalence and characteristics of ASD among children aged 8 years whose parents or guardians reside in 11 ADDM Network sites in the United States (Arkansas, Arizona, Colorado, Georgia, Maryland, Missouri, New Jersey, North Carolina, South Carolina, Utah, and Wisconsin). For 2012, the combined estimated prevalence of ASD among the 11 ADDM Network sites was 14.6 per 1,000 (one in 68). Estimated prevalence was significantly higher among boys $(23.6$ per 1,000$)$ than among girls $(5.3$ per 1,000$)$. Estimated prevalence varied widely among the 11 ADDM Network sites, ranges from 8.2 per 1,000 (in the area of the Maryland site where only health care records were reviewed) to 24.6 per 1,000 (in New Jersey, where both education and health care records were reviewed).

UK: In 2009 study, Baron-Cohen and colleagues calculated prevalence of ASD in mainstream and special schools (in both the state and the private sectors) within the county of Cambridgeshire. They estimated the prevalence to be 157 per 10,000 (including previously undiagnosed cases). However, the authors say that results from this 
study should be treated with caution because of the low response and the finding that the socioeconomic distribution of the population was not representative of the UK population. Nevertheless, the studies like this suggest that appropriate services should plan to meet the needs of between $1-2 \%$ of the primary school-aged population. ${ }^{3}$ The Adult Psychiatric Morbidity Survey, ${ }^{4}$ indicates that around $1 \%$ of the adult population in England is autistic, reaffirming the findings from the last survey in 2007. (However, the data does not include people who live in residential care). The recommendation of all these surveys is that the government, the NHS and local authorities have a responsibility to the 1 in 100 people who are autistic to make sure that they can get an early diagnosis and support and that the appropriate services are available. The National Autistic Society (2016) reports that there are thousands of autistic people and families each year who are in desperate need of help but aren't getting it.

Autism with learning disability: Emerson \& Baines, ${ }^{5}$ summarise the data from several countries about the prevalence of learning disabilities among autistic children in studies since 2000 till 2010: UK: from $15 \%$ to $71 \%$; USA - $37 \%$; Iceland - $84 \%$; Finland - $50 \%$. The average across the reviewed studies is $52.6 \%$. Compared to children with ASD alone, those with comorbid ID exhibited increased symptom severity and distinct DSM-IV-TR profiles. ${ }^{6}$ Christensen's, ${ }^{7}$ recommendations include enhancing strategies to lower the age of first evaluation of ASD by community providers: by 2020, children with ASD to be evaluated by age 36 months and begin receiving community-based support and services by age 48 months. The NAS and other non-governmental organisations use the prevalence figures to campaign for the Government, NHS and councils to make sure services are available for the right number of people, and to bring down diagnosis waiting time and for every area to have the services that autistic children need.

\section{Diagnosis}

\section{In search of biomarkers}

Many psychiatric disorders (including autism) are based on a descriptive collection of behaviours as no objective tests are available. There are no medical tests to diagnose autism yet, although soon it may change. Abi-Dargham A \& Horga $G{ }^{8}$ suggest steps toward the development of robust, reliable and valid biomarkers in order "to enable the field of psychiatry to move forward into the era of modern medicine." They suggest that neuroimaging techniques such as molecular imaging with positron-emission tomography (PET) or structural and functional magnetic resonance imaging (MRI) provide an opportunity to find biomarkers for different psychiatric disorders and conditions. Zaman, et al. ${ }^{9}$ have found a link between autism and the lack of an antibody called immunoglobulin G type 1, or IgG1 and urge to research further in order to develop blood test that can reveal autism. Parallel to research of biological markers, there is a lot of studies aiming to identify the behavioural symptoms that can indicate autism in very young babies (in contrast to social and communication symptoms which are not visible till 3 or 4 years), because the sooner we start supporting these infants, the better outcome may be for them. Most of these new studies research sensory perceptual 'symptoms' (behaviours), e.g., Cheung, et al. ${ }^{10}$ demonstrate that atypical perception and core ASD symptoms of social interaction and communication are closely and selectively associated during early development, and suggest causal links between perceptual and social features of ASD.

Since the first official description of autism in 1943 the diagnosis of the condition (and its name) has been changing. The latest change was in 2013, when the fifth edition of DSM-V was published. This change has created come problems for the comparative research studies because some of those who were diagnosed with ASD under DSMIV, wouldn't meet autism criteria under DSM-V. ${ }^{11}$ Any comparison of treatments and outcomes may be confusing at best and misleading at worst. E.g., Jashar, et al. ${ }^{11}$ compared adaptive and cognitive skills, and autism severity of toddlers with an ASD diagnosis under DSM-IV but not DSM-5 criteria to those who met autism criteria under both diagnostic systems (DSM-5 group) and to those without ASD (nonASD group). The toddlers in the DSM-IV only group were less delayed on various domains of adaptive (Communication, Socialization) and cognitive (Expressive and Receptive language, Fine Motor, Visual Reception) skills, and had less severe symptoms of ASD than the DSM-5 group. Thus, they might have the best potential for successful intervention. The DSM-IV only group did not differ from the nonASD group in any adaptive or cognitive skills except for socialization skills, the hallmark of ASD." Another problem is, different specialists use different diagnostic tools even in one and the same country. Here is just one example:

Australia: though there are specific diagnostic services available through the country, there is currently no agreement on Australian standard for the diagnosis of ASD. Taylor, et al..$^{12}$ assessed the diagnostic practices of health professionals across the nation (hospitals, nongovernmental organisations, publicly funded diagnostic services and private practice) and reported that while some clinicians work within a multidisciplinary assessment team, other practice independently and rarely collaborate with other clinicians to make a diagnostic decision. Only half said that they include a standardised objective assessment tool such as the Autism Diagnostic Schedule in ASD assessments, and one-third indicated that they do not include measures of development, cognition and language in assessments where ASD is suspected. The authors concluded that reported practice of some professionals in Australia may not be consistent with international best practice guidelines for ASD diagnosis and there is the need for a minimum national standard for ASD diagnosis throughout the country that ensures best practice regardless of the type of setting in which the service is provided.

It is important to remember that autism affects not only the child with the condition but the whole family. The news that the child is autistic is sometimes described as an 'autism bomb'; the devastation is considerable. In the UK, the NAS is one of the first contact points for the family. They provide valuable information and advice what services are available in the area. The social services become involved, they help the family to find the right educational setting for the child and provide respite care for the parents.

\section{Comorbids}

Quite often autistic individuals have other medical problems as well. Recent research has revealed specific medical conditions often associated with ASD, e.g. allergic asthma. ${ }^{13}$ Zachor \& Ben-Itzchak, ${ }^{14}$ investigated whether such medical conditions as macrocephaly, microcephaly, developmental regression, food selectivity, and sleep problems in ASD are associated with unique behavioral profiles. Based on their findings, they suggested two unique medical-behavioral subtypes in ASD that affect inherited traits of cognition and/or autism:

a. The microcephaly phenotype occurred with more impaired cognition and the developmental regression phenotype with widespread, more severe impairments in cognition and autism severity. 
b. In contrast, severe food selectivity and sleep problems represent only comorbidities to ASD that affect functioning. The researchers suggest that defining specific subgroups in ASD with a unique biological signature and specific behavioral phenotypes may help future genetic and neuroscience research.

\section{Children at risk}

Another development worth considering is assessment of 'children at risk' and providing services and professional consultation as soon as possible, and not waiting for a diagnostic assessment (wasting valuable time). With early support many of these children won't need a diagnosis and will adjust to the environment without any problems. Walton, ${ }^{15}$ examined risk factors for behavioural and emotional problems in siblings of children with ASD, revealing "six correlates: male gender, smaller family size, older age of the child with ASD, lower family income, child with ASD behavior problems, and sibling Broader Autism Phenotype. Siblings with few risk factors were at low risk for behavioral and emotional problems. However, siblings with many risk factors were at increased risk for both internalizing and externalizing problems." An interesting approach to such children has been developed in Japan, when the number of schoolchildren with undiagnosed developmental disorders has risen. Healthcare professionals call them 'kininaru-kodomo' (KK, "children of concern"); they are often overlooked at infant medical checkups, leaving them without necessary medical care and special needs education. Ide-Okochi \& Tadaka ${ }^{16}$ explore the KK concept "to enable professionals to properly assess and provide for the healthcare needs of these children at a high risk of DD, ideally with early intervention." The authors classified KK children into three groups:

a. Children who require special care.

b. Children whose special healthcare needs are owing to both individual and environmental factors.

c. Children waiting for the development of a new support system for them or their parents - all need support even before diagnosis.

\section{Interventions}

\section{Early interventions}

The National Autistic Society in the UK runs a number of programmes for families of children on the autism spectrum, such as, for example, Early Bird Programme (under 5 years) and Early Bird Plus (ages 4-8). They aim to support parents in the period between diagnosis and school placement. They combine group training sessions for parents with individual home visits when video feedback is used to help parents to establish interaction and communication with their child and managing behaviours. It is also important include "motor programming as part of the early intervention services delivered to young children with autism spectrum" that produce beneficial effect for the child development. ${ }^{17}$ There are hundreds of therapies for autism, and every year more new therapies spring to life. Some will work for some children while showing no benefit for, or even harming others. No two autistic children are alike. We should be very careful applying one and the same approach to all the children in the classroom, for example, using visual aids are very good for visual thinkers, but useless or even confusing for those whose thinking in 'sound images' or 'tactile pictures'.

\section{Educational provisions}

A range of educational provision exists, most are funded by authorities, but some schools are run by independent organisations. In the UK, these are:

a. Mainstream schools with or without extra support.

b. Generic special schools or units for students with learning disabilities.

c. Schools, units or classes which are specific to autism.

d. Schools or units for children with other types of special educational needs (e.g., sensory or physical difficulties, language problems, emotional and behavioural problems).

e. Residential schools.

f. Home-based programmes.

g. Outreach teams.

The majority of children with ASD attend schools or units within their local community, but some are provided placements in other parts of the country. It sounds quite good, but in reality, there are still many problems, for example:

a. $34 \%$ of children on the autism spectrum say that the worst thing about being at school is being picked on.18

b. $63 \%$ of children on the autism spectrum are not in the kind of school their parents believe would best support them.18

c. $17 \%$ of autistic children have been suspended from school; $48 \%$ of these had been suspended three or more times; $4 \%$ had been expelled from one or more schools. 18

\section{Adults with ASD}

The first generation of diagnosed patients with ASD is now in old age. Due to death of their parents and loss of social structure and support will need the medical community to help them. ${ }^{19}$ Now it's expected that most (all?) cases of ASD are diagnosed in early childhood. However, there are many undiagnosed adults with ASD who may have met the current diagnostic criteria for ASD when they were children but were either misdiagnosed or not diagnosed at all (in case of HFA - less impairing phenotypes) at the time because the diagnosis of ASD did not exist. Those who missed a formal diagnosis in childhood often seek a formal diagnosis later in life as in old age the symptoms are likely to worsen. Though there have been suggestions that symptoms may reduce with aging (e.g., ${ }^{20}$ - with 34 adults), the latest research $\left({ }^{21}\right.$ - with 100 adults) provides evidence age and severity of autism are linked, i.e. as age increases so does the severity of autism symptoms in social situations, communication and flexible thinking. (However, reporting more symptoms could also reflect a change in self-awareness. Better self-awareness is generally a good thing, but might lead to greater realisation of one's own difficulties).

In their study, Happé F, et al..$^{21}$ describes those with autism who have cognitive abilities in the normal range, i.e. with HFA. Despite this and the fact that they have developed strategies to help them function, older people in the study showed more severe symptoms of autism. Of course, ageing may also be different for each person with autism, and more research is needed to understand how autistic people change as they get older. Nevertheless, they all need help and 
services to support them now. $70 \%$ of autistic adults say that they are not getting the help they need from social services. Seventy per cent of autistic adults also told us that with more support they would feel less isolated..$^{22}$ At least one in three autistic adults are experiencing severe mental health difficulties due to a lack of support. ${ }^{23}$ Only $16 \%$ of autistic adults in the UK are in full-time paid employment, and only $32 \%$ are in some kind of paid work. Overall, only $32 \%$ of autistic adults are in some kind of paid work. 4 in 10 working parttime want to work more hours. ${ }^{24}$ Only $10 \%$ of autistic adults receive employment support but 53\% say they want it. ${ }^{22}$ And last but not least: Heterogeneity (Not autism, but autisms).

Though autism is a discrete clinical diagnosis, autistic traits exist on a continuum, extending into the general population. Ruzich, et al..$^{25}$ measure the distribution of autistic traits in siblings of individuals with autism. Their findings confirm the broader autism phenotype in siblings. More and more research studies have been published recently that emphasise the existence of subgroups of ASD with different underlying mechanisms (though the behavioural manifestation is the same. In order to obtain a deeper understanding of ASD, different (and more homogenous) subgroups should be defined (e.g. ${ }^{26,27}$ ). Thus, ASD is a complex neurodevelopmental disorder which is characterized by marked heterogeneity. ${ }^{28}$ Brian, et al. ${ }^{28}$ review recent research advances in genetics and neuroimaging, behavioural and psychopharmacological treatment approach. Despite considerable complexity, patterns are beginning to emerge that can inform the identification of novel treatment targets and approaches. ${ }^{28}$ Heterogeneity poses a major problem to current research. To elucidate neurocognitive and aetiological underpinning of sociocognitive processes and inform clinical advances we need theoretical and empirical refinements. ${ }^{29-32}$

On the other hand, discovering heterogeneity is an exciting new development that can bring us closer to the understanding of 'autisms' of different children and the ways we can help them. There are lots of positive developments in awareness, research, diagnosis and practice aimed to improve support and services for people with ASD - both children and adults. However, there are many challenges ahead, that need united efforts from all those for whom autism is part of their life.

\section{Acknowledgements}

None.

\section{Conflict of interest}

The authors declare that there is no conflict of interests regarding the publication of this paper.

\section{References}

1. Grinker RR. Unstrange minds: Remapping the world of autism. J Clin Invest. 2007;117(12):3597.

2. Buescher AV, Cidav Z, Knapp M, et al. Costs of autism spectrum disorders in the United Kingdome and the United States. JAMA Pediatr. 2014;168(8):721-728.

3. Baron-Cohen S, Scott FJ, Allison C, et al. Prevalence of autism-spectrum conditions: UK school-based population study. $\mathrm{Br} J$ Psychiatry. 2009;194(6):500-509.

4. Brugha T, Spiers N, Bankart J, et al. Autism Spectrum Disorder. In: McManus S, Bebbington P, et al. editors. Adults Psychiatric Morbidity Survey: Survey of Mental Health and Wellbeing, Health and Social Care Information Centre. England, UK: 2016.
5. Emerson E, Baines S. Estimated Prevalence of Autism among Adults with Learning Disabilities in England. Public Health, England, UK: 2010. p. $1-18$.

6. Tonnsen BL, Boan AD, Bradley CC, et al. Prevalence of Autism Spectrum Disorders Among Children With Intellectual Disability. Am J Intellect Dev Disabil. 2016;121(6):487-500.

7. Christensen DL, Baio J, Naarden Braun K van, et al. Prevalence and characteristics of autism spectrum disorder among children aged 8 years - Autism and Developmental Disabilities Monitoring Network, 11 sites, United States, 2012. Surveillance Summaries. 2016;65(3):1-23.

8. Abi-Dargham A, Horga G. The search for imaging biomarkers in psychiatric disorders. Nat Med. 2016;22(11):1248-1255.

9. Zaman S, Yazdani U, Deng Y, et al. A search for blood biomarkers for autism: Peptoids. Scientific Repots. 2016;6:19164.

10. Cheung $\mathrm{CH}$, Bedford R, Johnson $\mathrm{MH}$, et al. Visual search performance in infants associates with later ASD diagnosis. Dev Cogn Neurosci. 2016;:S1878-9293(16):30121-30129.

11. Jashar DT, Brennan LA, Barton ML, et al. Cognitive and Adaptive Skills in Toddlers Who Meet Criteria for Autism in DSM-IV but not DSM-5. $J$ Autism Dev Disord. 2016;46(12):3667-3677.

12. Taylor LJ, Eapen V, Maybery MT, et al. Diagnostic evolution for autism spectrum disorders: a survey of health professionals in Australia. BMJ Open. 2016;6(9):e012517.

13. Tonacci A, Billeci L, Ruta L, et al. A systematic review of the association between allergic asthma and autism. Minerva Pediatr. 2017;69(6):538-550.

14. Zachor DA, Ben-Itzchak E. Specific Medical Conditions Are Associated with Unique Behavioral Profiles in Autism Spectrum Disorders. Front Neurosci. 2016;10:410.

15. Walton KM. Risk Factors for Behavioral and Emotional Difficulties in Siblings of Children With Autism Spectrum Disorder. Am J Intellect Dev Disabil. 2016;121(6):533-549.

16. Ide-Okochi A, Tadaka E. A hybrid concept analysis of children of concern: Japanese healthcare professionals' views of children at a high risk of developmental disability. BMC Pediatr. 2016;16(1):171.

17. Ketcheson L, Hauck J, Ulrich D. The effects of an early motor skill intervention on motor skills, levels of physical activity, and socialization in young children with autism spectrum disorder: A pilot study. Autism. 2017;21(4):481-492.

18. Reid B, Ayris L ed. We've got great expectations: the chance of a lifetime for children with autism. London, UK, National Autistic Society, 2011.

19. Hategan A, Bourgeois JA, Goldberg J. Aging with autism spectrum disorder: an emerging public health problem. Int Psychogeriatr. 2017;29(4):695-697.

20. Fein D, Barton M, Eigsti IM, et al. Optimal outcome in individuals with a history of autism. J Child Psychol Psychiatry. 2013;54(2):195-205.

21. Happé FG, Mansour H, Barrett P, et al. Demographic and cognitive profile of individuals seeking a diagnosis of Autism Spectrum Disorder in adulthood. J Autism Dev Disord. 2016;46(11):3469-3480.

22. Bancroft K. The way we are: autism in 2012. London, UK, National Autistic Society, 2012. 40 p.

23. Rosenblatt M. I exist: message from adults with autism in England. London, UK, National Autistic Society, 2008. 46 p.

24. NAS. The autism employment gap: too much information in the workplace. London, UK, National Autistic Society, 2016. 24 p. 
25. Ruzich E, Allison C, Smith P, et al. The Autism-Spectrum Quotient in Siblings of People with Autism. Autism Res. 2016;10(2):289-297.

26. Bryn V, Aass HS, Skjeldal OH, et al. Cytokine Profile in Autism Spectrum Disorders in Children. J Mol Neurosci. 2017;61(1):1-7.

27. Martinez-Murcia FJ, Lai MC, Gorriz JM, et al. On the brain structure heterogeneity of autism: Parsing out acquisition site effects with significance-weighted principal component analysis. Hum Brain Mapp. 2017;38(3):1208-1223.

28. Brian J, Doyle-Thomas KAR, Baribeau D, et al. Novel mechanisms and treatment approaches in autism spectrum disorder. Discov Med. 2016;22(119):47-54.
29. Happé F, Conway JR. Recent progress in understanding skills and impairments in social cognition. Curr Opin Pediatr. 2016;28(6):736-742.

30. Baxter AJ, Brugha TS, Erskine HE, et al. The epidemiology and global burden of autism spectrum disorders. Psychol Med. 2015;45(3):601-613.

31. Kanner L. Autistic disturbances of affective contact. Nervous Child. $1943 ; 2: 217-250$

32. NAS. What do today's autism prevalence figures mean? London, UK, National Autistic Society, 2016. 